\title{
ON THE INCLINATION AND HABITABILITY OF THE HD 10180 SYSTEM
}

\author{
Stephen R. Kane ${ }^{1}$ and Dawn M. Gelino ${ }^{2}$ \\ ${ }^{1}$ Department of Physics and Astronomy, San Francisco State University, 1600 Holloway Avenue, San Francisco, CA 94132, USA; skane@ sfsu.edu \\ ${ }^{2}$ NASA Exoplanet Science Institute, Caltech, MS 100-22, 770 South Wilson Avenue, Pasadena, CA 91125, USA \\ Received 2014 March 11; accepted 2014 July 28; published 2014 August 21
}

\begin{abstract}
There are numerous multi-planet systems that have now been detected via a variety of techniques. These systems exhibit a range of both planetary properties and orbital configurations. For those systems without detected planetary transits, a significant unknown factor is the orbital inclination. This produces an uncertainty in the mass of the planets and their related properties, such as atmospheric scale height. Here we investigate the HD 10180 system, which was discovered using the radial velocity technique. We provide a new orbital solution for the system which allows for eccentric orbits for all planets. We show how the inclination of the system affects the mass/radius properties of the planets and how the detection of phase signatures may resolve the inclination ambiguity. We finally evaluate the Habitable Zone properties of the system and show that the g planet spends $100 \%$ of an eccentric orbit within the Habitable Zone.
\end{abstract}

Key words: astrobiology - planetary systems - stars: individual (HD 10180)

\section{INTRODUCTION}

Multi-planet system discoveries have revealed a diversity of system architectures, many of which significantly diverge from that of our own solar system. Many of the recent multi-planet system discoveries have been made as a result of data from the Kepler mission, such as Kepler-62 (Borucki et al. 2013). These systems tend to harbor a mixture of terrestrial and Neptune-size planets, some of which are in the Habitable Zone (HZ) of their host star. There have also been several discoveries of systems with more than four planets that have been discovered by radial velocity (RV) surveys, such as the 55 Cancri system (McArthur et al. 2004; Endl et al. 2012). The orbital inclination of the planets in these cases is generally unknown, although they can be constrained through examination of the dynamical stability of the system (e.g., Correia et al. 2010).

A multi-planet system of particular interest is the HD 10180 system, due to the both the relatively large number of planets and their relatively low masses. There have been various interpretations of the RV data for this system with respect to the number of planets present. Lovis et al. (2011) provide a seven planet solution where the detection of the inner "b" planet is considered tentative. A further solution by Tuomi (2012) demonstrates that the system may harbor nine planets. Although in both cases the planets likely have low masses, this depends on the inclination of the system with respect to the plane of the sky. As the inclination decreases the mass of the planets increases and thus their physical properties change. The inclination ambiguity can be resolved using several techniques, such as astrometry (Tuomi et al. 2009) and phase curve analysis (Kane \& Gelino 2011, 2012a). Determining the true masses of the planets is a key factor in determining the significance of their locations within the stellar HZ (Kopparapu et al. 2014).

Here we present the results of a new analysis of the HD 10180 system in which we discuss the orbital parameters, inclination, predicted phase signatures, and $\mathrm{HZ}$ status of the planets. In Section 2, we provide a new Keplerian orbital solution for the system with eccentric orbits for all planets. Section 3 investigates the effects of orbital inclination on planet masses and possible radii. Section 4 discusses the phase variations of the system in different inclination scenarios and the detectability of those signatures. In Section 5, we present an analysis of the system HZ in the context of various inclinations. We provide concluding remarks in Section 6.

\section{SYSTEM CONFIGURATION}

HD 10180 is a star which is quite similar to solar (G1V) in terms of its fundamental properties. These are summarized in Table 1 , where the majority of parameters are those provided by Lovis et al. (2011). The distance is derived from Hipparcos parallax measurements (van Leeuwen 2007) and the stellar radius is calculated from the mass-radius relationships determined by Torres et al. (2010). As noted by Lovis et al. (2011), the activity index shows that HD 10180 is a relatively inactive star, a property that will be of particular relevance when discussing the photometry in Section 4.

The Keplerian orbital solution provided by Lovis et al. (2011) includes seven planets and forces a circular orbit for several of those planets, including planet g. Since the semiamplitude of the RV signal for the $b$ planet is significantly lower than the others, we performed our own fit to the RV data to obtain a Keplerian orbital solution in which all eccentricities were allowed to vary as free parameters. The RV data were extracted from the VizieR Catalog Service. ${ }^{3}$ These consist of 190 measurements obtained with the HARPS spectrograph at the ESO $3.6 \mathrm{~m}$ telescope at La Silla Observatory. We fit the data using the partially linearized, least-squares fitting procedure described in Wright et al. (2009) and estimated parameter uncertainties using the BOOTTRAN bootstrapping routines described in Wang et al. (2012). Our best solution includes six planets where there is no significant RV trend in the data. We adopted a slightly larger stellar jitter value than Lovis et al. (2011) of $1.39 \mathrm{~m} \mathrm{~s}^{-1}$ which forces the reduced $\chi^{2}$ value to unity. The resulting orbital solution is shown in Figure 1 and Table 2 . The residuals shown in the bottom panel of Figure 1 have an rms scatter of $1.5 \mathrm{~m} \mathrm{~s}^{-1}$. The main differences with the solution by Lovis et al. (2011) are (1) no planet b, (2) a significant eccentricity for planet g, and (3) a smaller orbital period and eccentricity for planet h. Note that Lovis et al. (2011)

\footnotetext{
3 http://vizier.u-strasbg.fr/
} 

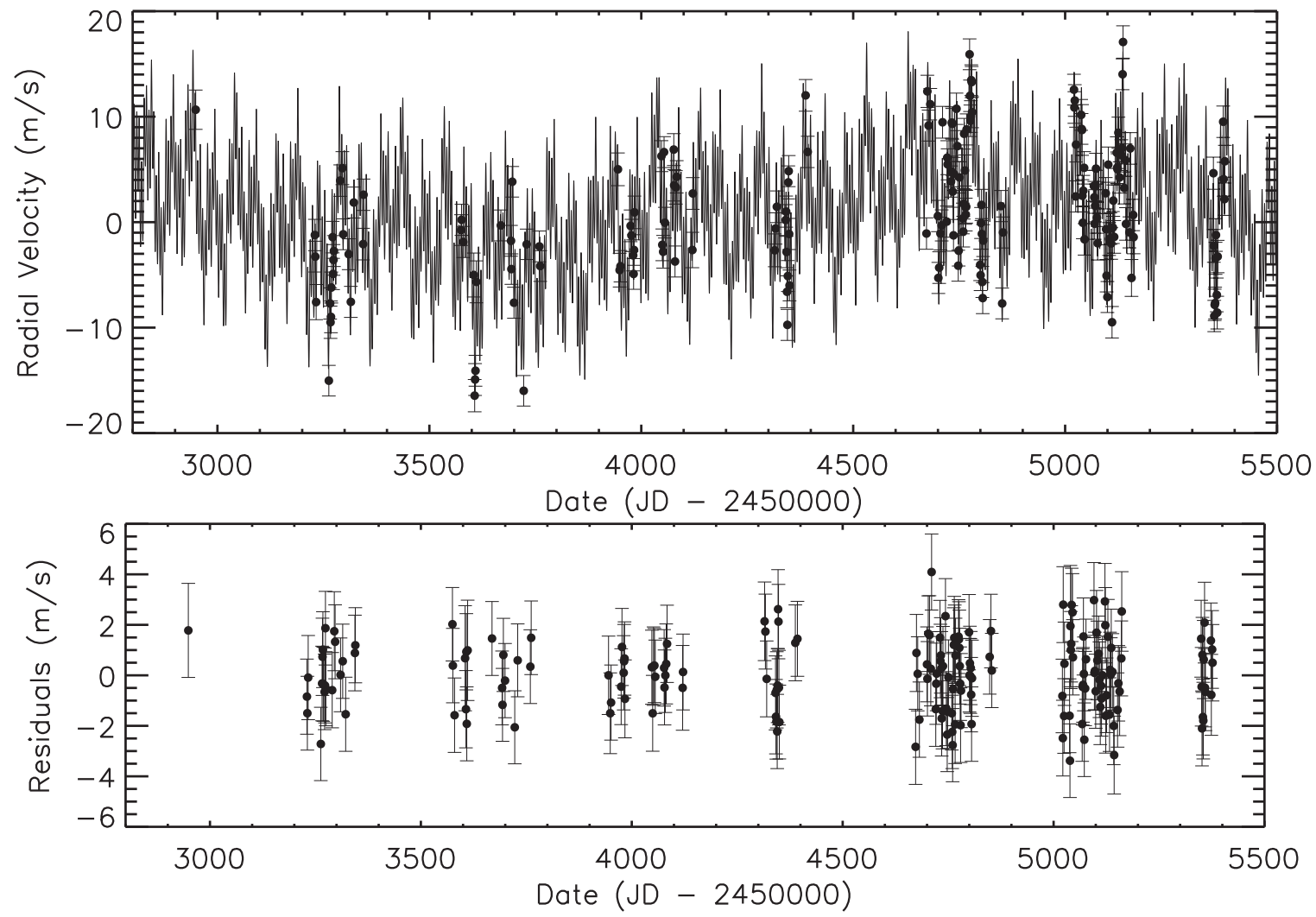

Figure 1. Top panel: the 190 RV measurements of HD 10180 along with the best-fit six-planet Keplerian solution. The solution, shown in Table 2, allows the eccentricities of all planets to be free parameters. Bottom panel: the RV residuals (observed minus computed) from the best-fit model shown above.

Table 1

Stellar Parameters ${ }^{\mathrm{a}}$

\begin{tabular}{lc}
\hline \hline Parameter & Value \\
\hline$V$ & 7.33 \\
$B-V$ & 0.629 \\
Distance $(\mathrm{pc})^{\mathrm{b}}$ & $39.02 \pm 1.1$ \\
$T_{\text {eff }}(\mathrm{K})$ & $5911 \pm 19$ \\
$\log g$ & $4.39 \pm 0.03$ \\
{$[\mathrm{Fe} / \mathrm{H}](\mathrm{dex})$} & $0.08 \pm 0.01$ \\
$M_{\star}\left(M_{\odot}\right)$ & $1.06 \pm 0.05$ \\
$R_{\star}\left(R_{\odot}\right)^{\mathrm{c}}$ & $1.109 \pm 0.036$ \\
\hline
\end{tabular}

Notes.

${ }^{\text {a }}$ Lovis et al. (2011).

b van Leeuwen (2007).

c Torres et al. (2010).

force the eccentricity of the $\mathrm{g}$ planet to zero since a non-zero eccentricity produces an almost identical $\chi^{2}$. Here we consider a non-zero eccentricity for the $g$ planet since it is consistent with the data and is relevant to our subsequent habitability discussion in Section 5.

Since this eccentric solution is different from those previously published, it is important to establish if it is dynamically sound. To explore this, we performed dynamical simulations using $N$-body integrations with the Mercury Integrator package, described in more detail by Chambers (1999). We adopted the hybrid symplectic/Bulirsch-Stoer integrator and used a Jacobi coordinate system. This coordinate system generally provides more accurate results for multi-planet systems (Wisdom \& Holman 1991; Wisdom 2006) except in cases of close encounters (Chambers 1999). The integrations were performed for a simulation of $10^{6} \mathrm{yr}$, in steps of $100 \mathrm{yr}$, starting at the present epoch.

Our simulations indicate that the orbital configuration presented in Table 2 are stable over the $10^{6} \mathrm{yr}$ simulation. The planets do exchange angular momentum through secular oscillations of their eccentricities, but this remains at a relatively low level. The two main examples of this are the outermost ( $\mathrm{g}$ and $\mathrm{h}$ ) planets. The eccentricity oscillations for both of these planets are shown in Figure 2 for the complete simulation period. The secular oscillations complete approximately 15 cycles during the $10^{6} \mathrm{yr}$ simulation with a period of $\sim 65,000 \mathrm{yr}$. The range of eccentricity for the $g$ and $h$ planets are $0.263-0.321$ and $0.042-0.095$, respectively. The g planet eccentricity oscillations are also perturbed due to interactions with the f planet. We discuss the implications of these oscillations further in Section 5.

\section{INCLINATION AND PLANETARY PROPERTIES}

There is a well-studied relationship between planetary mass and radius. Early work on mass-radius relationships for gas giants (Fortney et al. 2007) and super-Earths (Seager et al. 2007) paved the way for understanding the results of Kepler discoveries. Kepler planets have subsequently allowed empirical relations to be developed for low-mass planets (Weiss et al. 2013; Weiss \& Marcy 2014). The nature of RV discoveries of exoplanet that lack confirmation from other techniques is that it is only the minimum mass that is known. The true planetary masses depend on the inclination of the system, from edge-on $\left(i=90^{\circ}\right)$ to face-on $\left(i=0^{\circ}\right)$ with respect to the plane of the sky.

In Figure 3, we show the increase in the HD 10180 planet masses as a function of orbital inclination. The $f$ and $g$ planets 


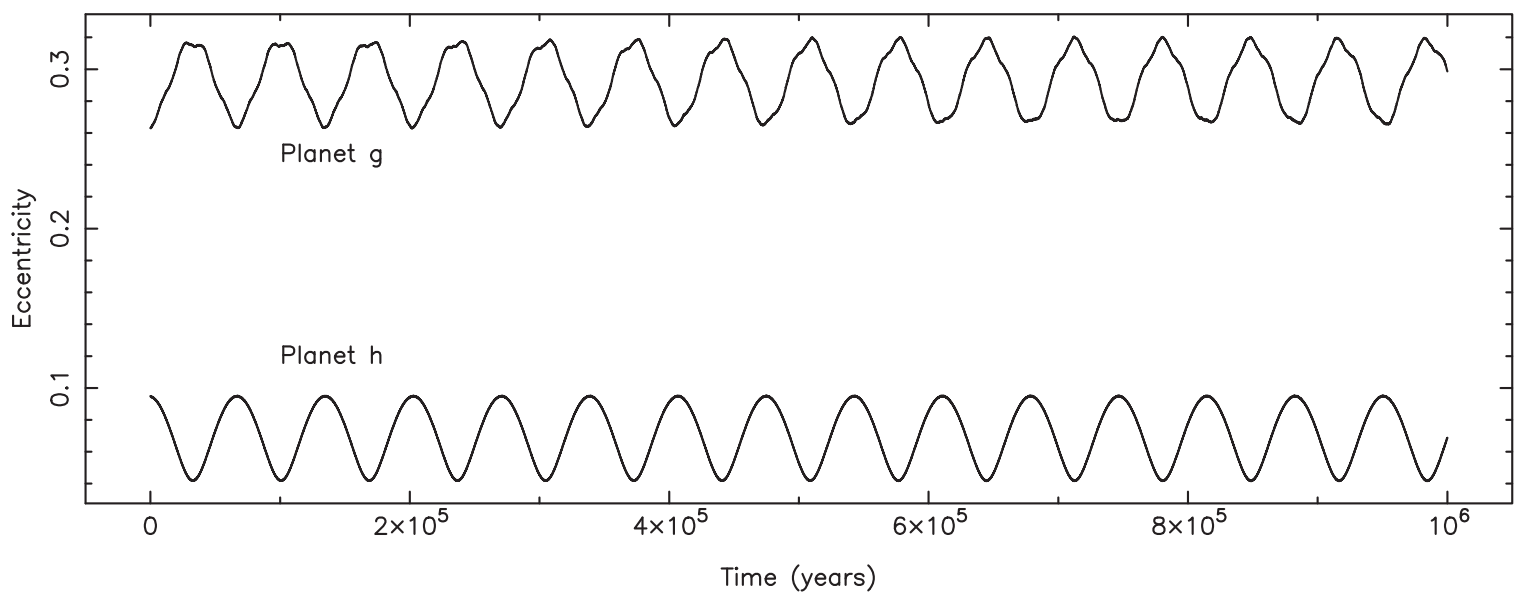

Figure 2. Dynamical simulations of the HD 10180 system, showing the eccentricity oscillations of the $\mathrm{g}$ and h planets over a period of $10^{6} \mathrm{yr}$. The g planet primarily dynamically interacts with the $h$ planet with additional minor perturbations caused by the f planet.

Table 2

HD 10180 Planetary Parameters

\begin{tabular}{|c|c|c|c|c|c|c|}
\hline Parameter & $c$ & $d$ & $e$ & $f$ & $g$ & $h$ \\
\hline$P$ (days) & $5.75969 \pm 0.00028$ & $16.3570 \pm 0.0038$ & $49.748 \pm 0.025$ & $122.744 \pm 0.232$ & $604.67 \pm 10.42$ & $2205.0 \pm 105.9$ \\
\hline$T_{p}^{\mathrm{a}}$ & $4001.445 \pm 0.426$ & $4022.119 \pm 1.157$ & $4006.26 \pm 6.91$ & $4024.67 \pm 11.03$ & $4002.8 \pm 68.3$ & $3433.4 \pm 393.1$ \\
\hline$e$ & $0.073 \pm 0.031$ & $0.131 \pm 0.052$ & $0.051 \pm 0.033$ & $0.119 \pm 0.054$ & $0.263 \pm 0.152$ & $0.095 \pm 0.086$ \\
\hline$\omega(\operatorname{deg})$ & $328 \pm 24$ & $325 \pm 23$ & $147 \pm 54$ & $327 \pm 27$ & $327 \pm 59$ & $142 \pm 72$ \\
\hline$K\left(\mathrm{~m} \mathrm{~s}^{-1}\right)$ & $4.545 \pm 0.154$ & $2.935 \pm 0.173$ & $4.283 \pm 0.169$ & $2.862 \pm 0.186$ & $1.754 \pm 0.380$ & $3.117 \pm 0.245$ \\
\hline$M_{p} \sin i\left(M_{J}\right)$ & $0.0416 \pm 0.0014$ & $0.0378 \pm 0.0022$ & $0.0805 \pm 0.0032$ & $0.0722 \pm 0.0047$ & $0.0732 \pm 0.0138$ & $0.2066 \pm 0.0139$ \\
\hline$a(\mathrm{AU})$ & $0.06412 \pm 0.00101$ & $0.12859 \pm 0.00202$ & $0.2699 \pm 0.0043$ & $0.4929 \pm 0.0078$ & $1.427 \pm 0.028$ & $3.381 \pm 0.121$ \\
\hline
\end{tabular}

Note. ${ }^{\text {a }}$ BJD $-2,450,000$.

have similar masses and so their lines in the plots are almost indistinguishable. The dynamical analysis of the HD 10180 system by Lovis et al. (2011) shows that the system is still stable for $i=30^{\circ}$ but not for $i=10^{\circ}$, concluding that an instability transition occurs around $i \sim 20^{\circ}$. We tested this instability transition by repeating our stability analysis described in Section 2 for a variety of inclinations. We confirm that the system becomes unstable at $i \sim 20^{\circ}$ (shown as a vertical dashed line in Figure 3) with the ejection of the d planet, but the $\mathrm{g}$ planet remains stable despite the higher eccentricity. The range of $g$ planet eccentricities described in Section 2 remains the same until instability occurs. The bottom panel of Figure 3 shows the corresponding change in planet radius using the simple mass-radius relationship of Kane \& Gelino (2012b) which assumes an approximate Jupiter radius for masses larger than $0.3 M_{J}$. We discuss the implications of these mass/radius increases in the following sections.

\section{PHASE VARIATIONS}

A further means through which to constrain the inclination of the system is by the detection of phase variations. This technique was described in detail by Kane \& Gelino (2012a) where the amplitude of the phase variations depend on the planetary properties which vary with inclination (see Section 3). One aspect of the system that affects the ability to detect such phase signatures is the activity of the star. HD 10180 is known to be a relatively inactive star with a mean activity index of $\log R_{\mathrm{HK}}^{\prime}=-5.00$ (Lovis et al. 2011). We used publicly available data from the Hipparcos satellite to search for low-frequency photometric variations of HD 10180. Hipparcos acquired a total of 125 measurements spanning a period of 1184 days during the course of its 3 yr mission (Perryman et al. 1997; van Leeuwen 2007). These data are shown in Figure 4. The $1 \sigma \mathrm{rms}$ scatter of the $125 \mathrm{HD} 10180$ measurements is $0.013 \mathrm{mag}$, while the mean of the measurement uncertainties is 0.011 . Thus it is consistent with a photometrically stable star at the $1 \%$ level. A Fourier analysis of the Hipparcos data do not reveal any significant periodic signatures and indeed the data Nyquist frequency of 0.0528 days $^{-1}$ is slightly above the predicted period of the stellar rotation ( $\sim 24$ days). The Hipparcos data sampling is therefore unlikely to detected stellar rotation variability.

Although it is advantageous that the star is relatively quiet, the phase variations occur on a much lower level. We calculate the predicted phase variations of the system by adopting the formalism of Kane \& Gelino (2010). This formalism accounts for planetary size, orbital eccentricity, and the variation of geometric albedo with separation from the host star. The flux ratio of the planet to the host star is given by

$$
\epsilon(\alpha, \lambda) \equiv \frac{f_{p}(\alpha, \lambda)}{f_{\star}(\lambda)}=A_{g}(\lambda) g(\alpha, \lambda) \frac{R_{p}^{2}}{r^{2}},
$$

where $A_{g}$ is the geometric albedo, $g(\alpha, \lambda)$ is the phase function, $R_{p}$ is the planetary radius, and $r$ is the star-planet separation. The resulting flux variations of the system are shown in the top three panels of Figure 5 where we have calculated the variations for system inclinations of $90^{\circ}$ (edge-on), $30^{\circ}$, and $10^{\circ}$. In each panel the phase flux variations due to the individual planets are shown as solid lines and the combined variations are indicated by the dotted line. These are shown for one complete orbital phase of the outer planet. As described in Section 3, the inclination must be larger than $10^{\circ}$ in order to retain a stable orbital configuration for the system. 

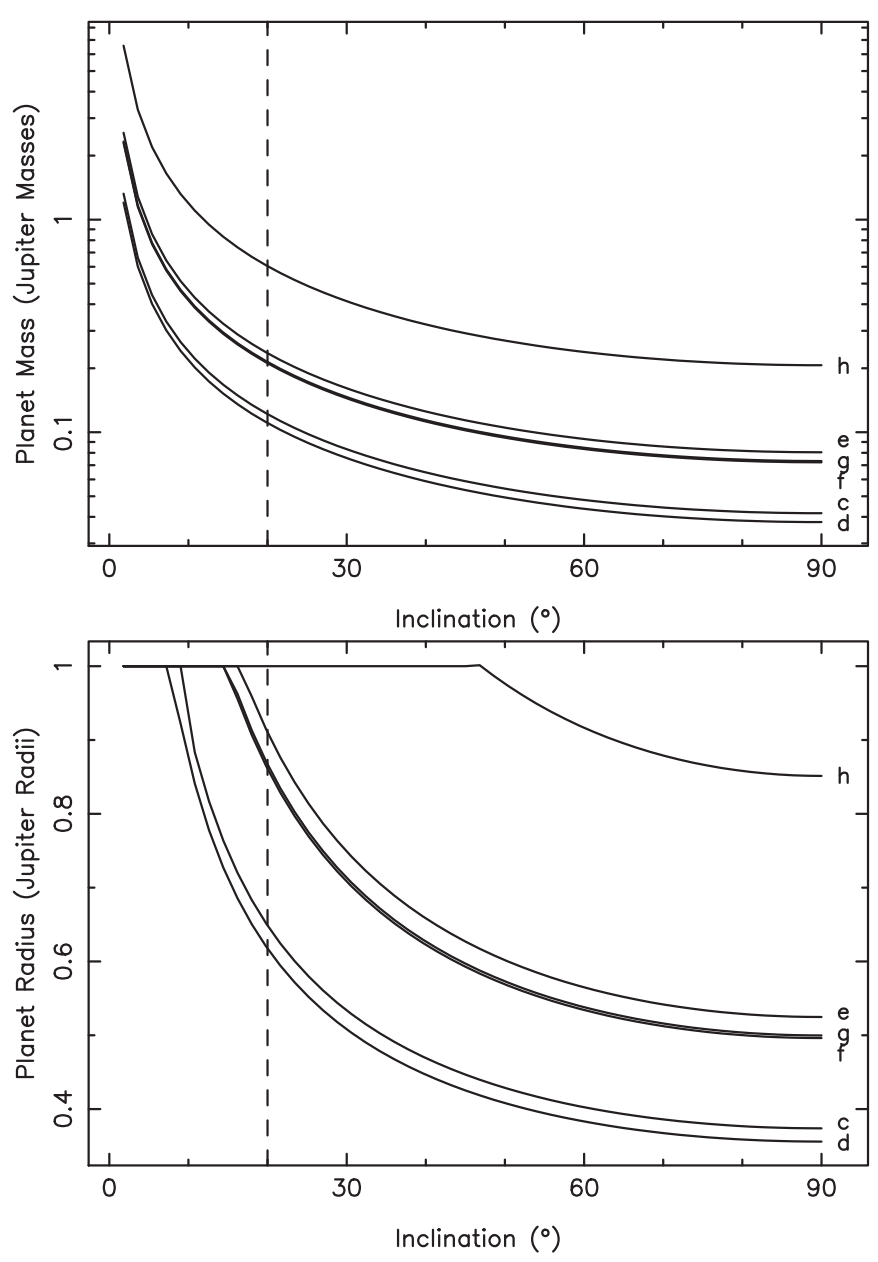

Figure 3. Dependence of the HD 10180 planetary properties of mass (top panel) and radius (bottom panel) on the system inclination, where $90^{\circ}$ is an edge-on orientation and $0^{\circ}$ is face-on. The vertical lines represent the likely lower inclination limit of the system as estimated by Lovis et al. (2011).

The phase variations of the $\mathrm{c}$ and d planets are also labeled on the right of each panel in Figure 5. The peak flux variations are dominated by the inner (c) planet for each inclination. The planet has a calculated radius of 0.37 and $0.92 R_{J}$ for inclinations of $90^{\circ}$ and $10^{\circ}$, respectively. As shown by Kane \& Gelino (2011), the effect of decreasing the inclination is to remove the time variability of the phase function resulting in flux variations caused exclusively by orbital eccentricity. This can be particularly seen for the $d$ planet in the bottom panel which retains a photometrically variable signature due to its eccentricity of 0.131 (see Table 2). The amplitude of the total variations remains very similar with decreasing inclination due to the compensation of the increased planetary radii. However, the constantly visible illumination of the $\mathrm{c}$ and $\mathrm{d}$ planets for lower inclinations significantly raises the baseline of the planetary reflected light received. The effect of this is to raise to signal-to-noise of the variations which makes their detection more accessible. A possible method to discriminate between the baseline flux from the planet and the stellar flux is through polarized light. The motion of the planet(s) will produce a polarization signature distinct from the stellar flux due to the scattering of light from the planetary atmospheres (Berdyugina et al. 2011; Wiktorowicz 2009). However, for Keplerian orbits an additional distinction is available via the difference in phase between the times of periastron and maximum phase variations (phase angle zero). The bottom panel of Figure 5 shows the difference between the combined flux variations of $i=10^{\circ}$ and $i=90^{\circ}$ after the minimum flux (baseline) has been removed. The $58^{\circ}$ separation of the periastron passage of the $\mathrm{c}$ planet from the zero phase angle produces a difference in phase signature of amplitude similar to the individual inclination scenarios. In practice, most systems will have an even larger separation which will aid in this distinction, the amplitude of which will depend on the orbital eccentricities. Resolving this degeneracy will greatly aid in disentangling the components of the phase signature and thus the inclination of the system. The total flux variations are of amplitude several parts per million and thus close to the photometric precision achieved by the Kepler mission for the brightest stars monitored. This technique could therefore be used to rule out low system inclinations and/or high albedos for multi-planet systems, particularly with data from future missions such as the James Webb Space telescope and the Transiting Exoplanet Survey Satellite.

\section{HABITABILITY OF THE g PLANET}

With the revised orbital solution of Section 2 and inclination effects described in Sections 3 and 4, we finally investigate the $\mathrm{HZ}$ of the system. The empirically derived HZ boundaries of Kasting et al. (1993) have recently been replaced by new calculations by Kopparapu et al. (2013, 2014). We use these calculations to provide new estimates for the HZ in the HD 10180 by adopting the definitions of "conservative" and "optimistic" HZ models described by Kane et al. (2013). These definitions use different boundaries for the $\mathrm{HZ}$ based on assumptions regarding the amount of time that Venus and Mars were able to retain liquid water on their surfaces (Kopparapu et al. 2013). Kane (2014)

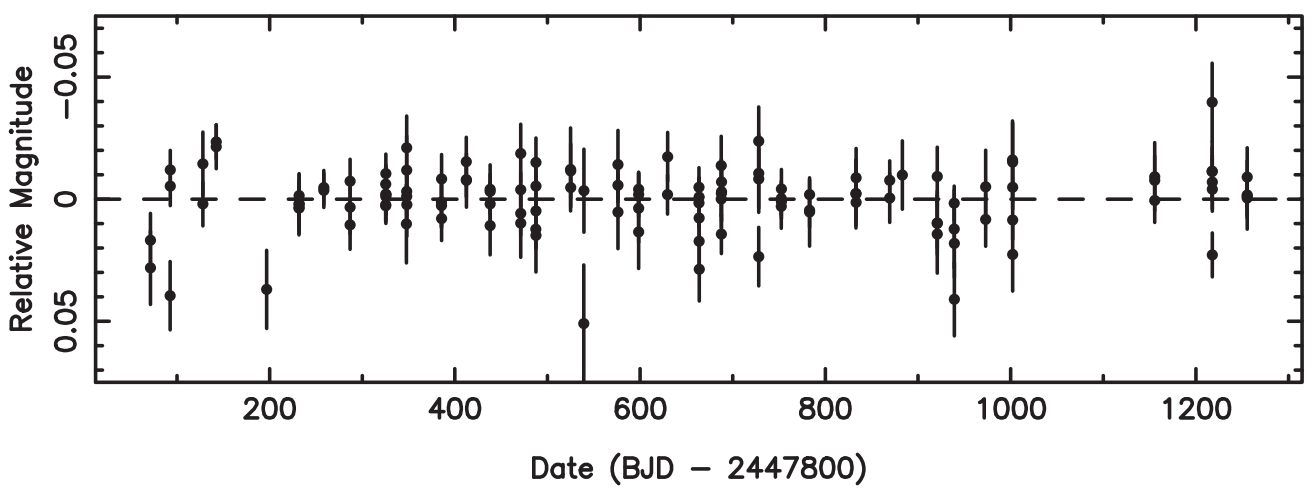

Figure 4. Photometry of HD 10180 from the Hipparcos mission that shows photometric stability at the $1 \%$ level. 

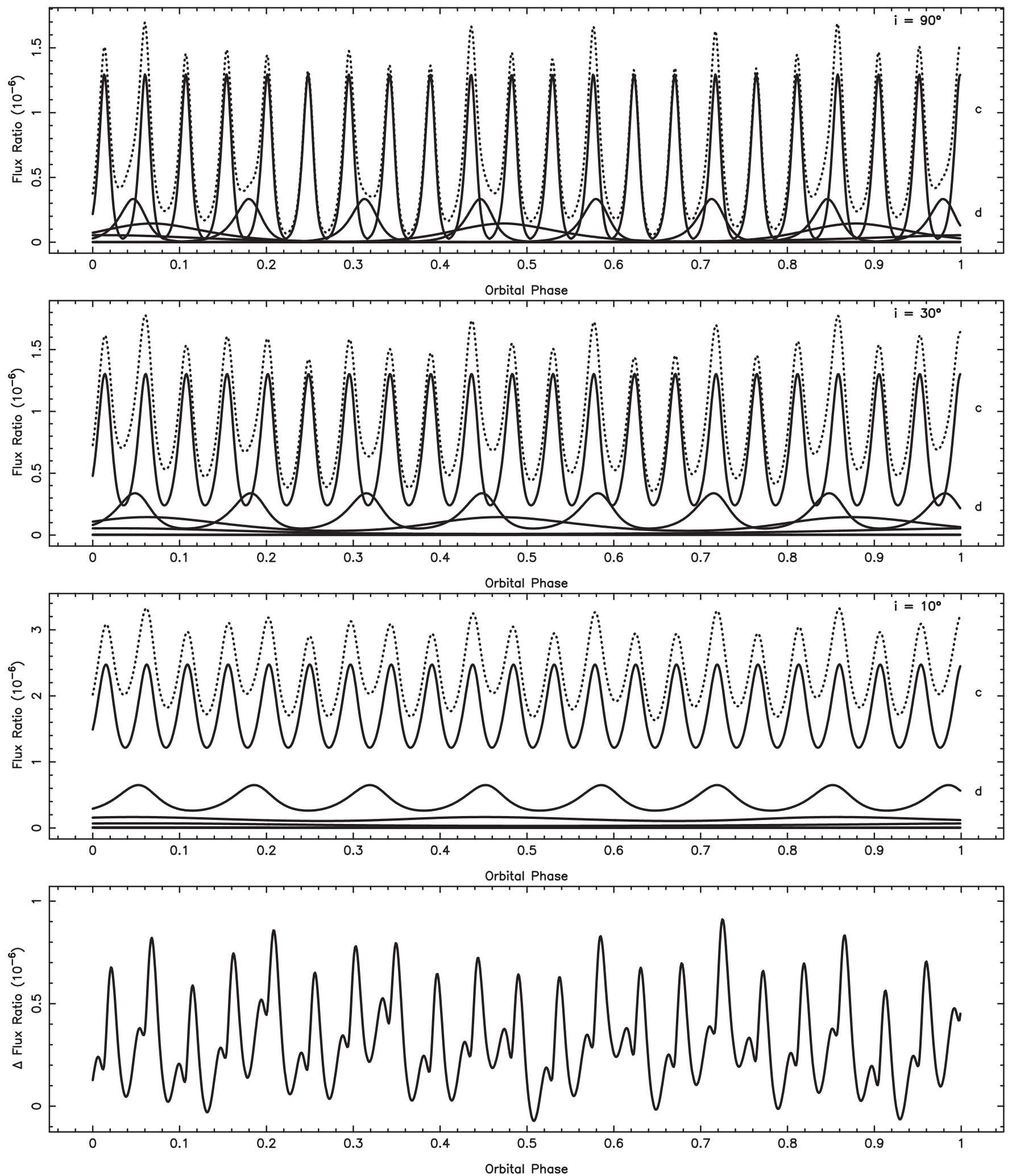

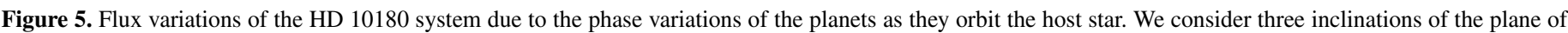

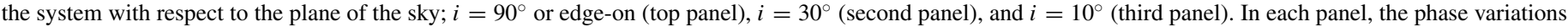

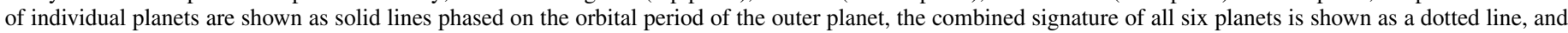

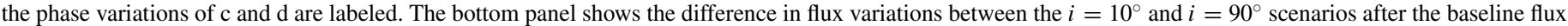
has been removed.

showed the extent to which HZ boundaries depend on stellar parameter uncertainties, although the parameters in Table 1 are sufficiently well known that the HZ boundary uncertainties are negligible. HZ calculations for all known exoplanetary systems are available using the same methodology through the Habitable Zone Gallery (Kane \& Gelino 2012b).

Figure 6 shows two top-down views of the HD 10180 system, one zoomed out to include the outer planet (left panel) and the 

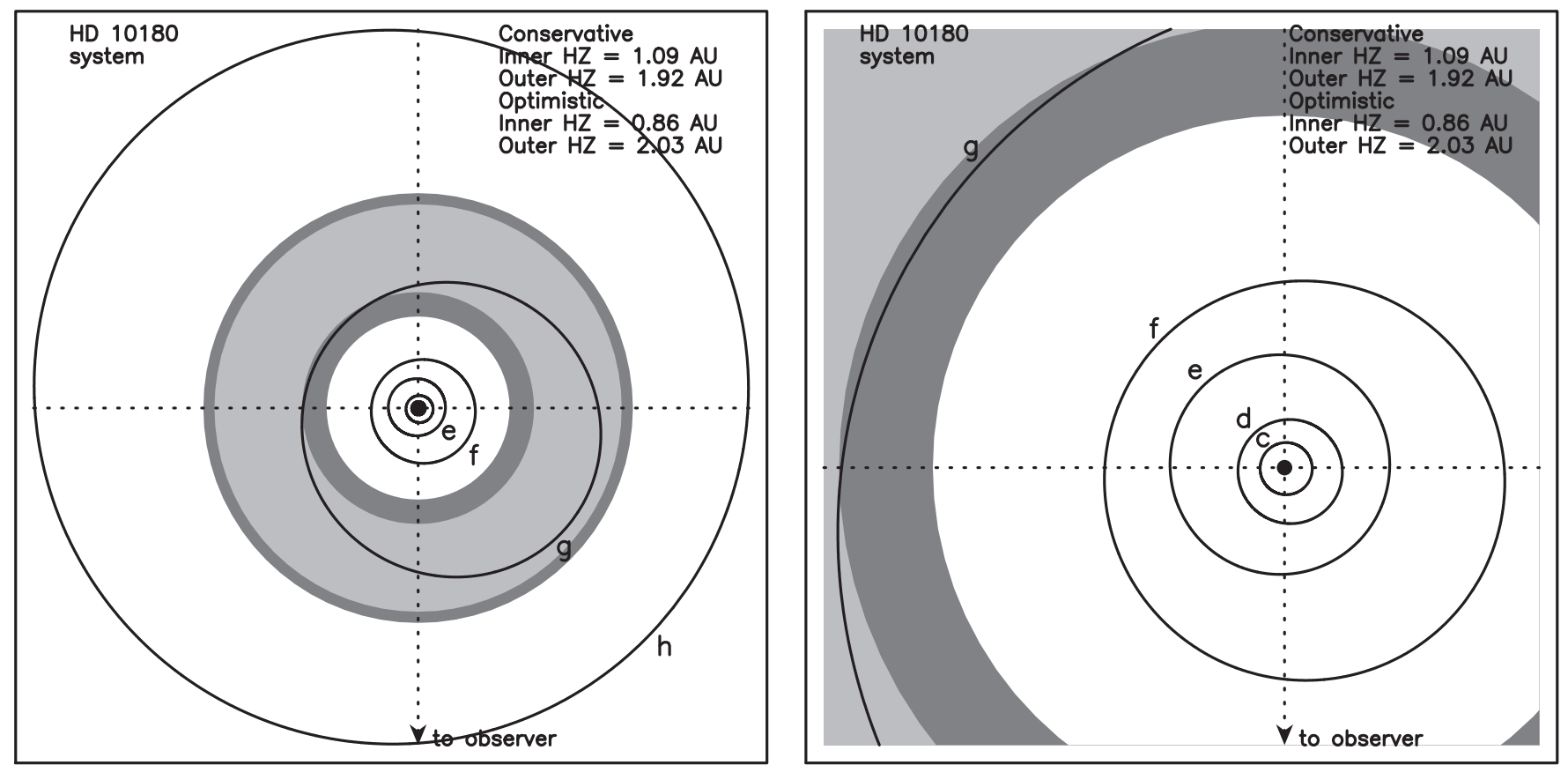

Figure 6. Top-down view of the HD 10180 system showing the extent of the $\mathrm{HZ}$ calculated using the stellar parameters of Table 1 . The conservative $\mathrm{HZ}$ is shown as light gray and optimistic extension to the $\mathrm{HZ}$ is shown as dark gray. The revised Keplerian orbits of the planets from Table 2 are overlaid. Left panel: The full $\mathrm{HZ}$ of the system with the outer five planets. Right panel: a zoom-in of the system showing the orbits of the inner five planets and the orbital path of the $g$ planet into the optimistic HZ.

other zoomed in to show the inner planets (right panel). In each panel, the HZ is depicted by the shaded region where the light gray represents the conservative $\mathrm{HZ}$ and the dark gray is the extension to the $\mathrm{HZ}$ with optimistic calculations. Of greatest interest in this figure from a HZ perspective is the g planet. The planet remains in the conservative $\mathrm{HZ}$ for $89 \%$ of the duration of one orbit with the remaining $11 \%$ within the optimistic HZ. The orbital path through the optimistic HZ occurs during the periastron passage and is clearly shown in the right panel of Figure 6. The orbital stability analysis from Section 2 showed that the eccentricity of the planet can oscillate to a value as high as $\sim 0.32$ over $\sim 65,000$ yr timescales. We recomputed our $\mathrm{HZ}$ calculations using this maximum eccentricity and found that the planet remains $100 \%$ in the HZ; $83 \%$ in the conservative HZ and $17 \%$ in the optimistic HZ. Thus interactions with the other planets do not perturb the HZ status of the planet. Note that increasing the eccentricity by the $1 \sigma$ uncertainty shown in Table $2(e=0.415)$ results in the planet passing slightly interior to the optimistic $\mathrm{HZ}$ during periastron passage, but it remains in the $\mathrm{HZ}$ for $94 \%$ of the orbital period. Planets in eccentric orbits which spend only part of their orbit in the HZ have been previously investigated (Kane \& Gelino 2012c; Williams \& Pollard 2002). These studies show that habitability is not necessarily ruled out depending on the efficiency of the planetary atmosphere in redistributing the variable energy from stellar insolation during the orbit.

For an inclination of $90^{\circ}$, the mass of the $g$ planet is $0.0732 M_{J}$ or $23.3 M_{\oplus}$. The estimated radius according to Figure 3 is $0.5 R_{J}$. An inclination of $30^{\circ}$ raises these values to $0.1464 M_{J}$, $46.5 M_{\oplus}$, and $0.71 R_{J}$, respectively. Adjusting the inclination to the stability limit of $10^{\circ}$ further raises the values to $0.422 M_{J}$, $134.0 M_{\oplus}$, and $1.0 R_{J}$, respectively. For any value of inclination, the mass of the $\mathrm{g}$ planet is far above the threshold where the planet is likely to have a purely rocky composition (Marcy et al. 2014). Thus the prospects for habitability for the g planet lies within a Moon system which the planet may harbor. Searches for exomoons around such HZ giant planets have been undertaken (Kipping et al. 2013) but have not yet yielded positive results. Recent studies of exomoon habitability have shown that there are a variety of factors which add to the total energy budget including flux from the planet and tidal effects (Heller 2012; Hinkel \& Kane 2013). Although these additional factors are usually negligible compared with the flux from the host star, they may be sufficient in this case to render the $g$ planet moons devoid of surface liquid water considering the planet already moves interior to the conservative HZ.

\section{CONCLUSIONS}

Multi-planet exoplanetary systems offer exceptional opportunities for system characterization, such as constraining the orbital inclinations and eccentricities based on stability simulations. These systems are particularly interesting when one or more of the planets occupy the HZ of their host star. The HD 10180 is just such a system, with a range of planetary masses and at least one planet within the HZ. We have presented a new orbital solution which allows the $\mathrm{g}$ planet to have significant orbital eccentricity whilst preserving the stability of the system. We have quantitatively shown how the properties of the planets alter depending on the inclination of the system. These properties in turn change the predicted phase signatures of the system and provides a method through which future observations could resolve the inclination ambiguity.

An important consideration for $\mathrm{HZ}$ planets is the orbital eccentricity since the variable stellar insolation can greatly effect the habitability. The revised orbital solution presented here allows for an eccentric orbit for the only HZ planet in the system. The HZ calculations described here show that the g planet spends most of a complete orbital period within the conservative $\mathrm{HZ}$ and moves into the interior optimistic $\mathrm{HZ}$ for 
the remaining time. The mass of the planet is high enough that surface liquid water is only possible on any moons the planet may possess. The topic of exomoons is one of increasing study and detection efforts and is thus likely to yield positive detections in the near future. As next-generation instrumentation is developed for exoplanetary studies, it is important to identify the best HZ targets orbiting bright host stars such as the one described here.

The authors thank the anonymous referee, whose comments greatly improved the quality of the paper. This research has made use of the following online resources: the Exoplanet Orbit Database and the Exoplanet Data Explorer at exoplanets.org, the Habitable Zone Gallery at hzgallery.org, and the VizieR catalog access tool, CDS, Strasbourg, France.

\section{REFERENCES}

Berdyugina, S. V., Berdyugin, A.V., Fluri, D. M., \& Piirola, V. 2011, ApJL, 728, L6

Borucki, W. J., Agol, E., Fressin, F., et al. 2013, Sci, 340, 587

Chambers, J. E. 1999, MNRAS, 304, 793

Correia, A. C.M., Couetdic, J., Laskar, J., et al. 2010, A\&A, 511, 21

Endl, M., Robertson, P., Cochran, W. D., et al. 2012, ApJ, 759, 19

Fortney, J. J., Marley, M. S., \& Barnes, J. W. 2007, ApJ, 659, 1661

Heller, R. 2012, A\&A, 545, L8

Hinkel, N. R., \& Kane, S. R. 2013, ApJ, 774, 27
Kane, S. R. 2014, ApJ, 782, 111

Kane, S. R., Barclay, T., \& Gelino, D. M. 2013, ApJL, 770, L20

Kane, S. R., \& Gelino, D. M. 2010, ApJ, 724, 818

Kane, S. R., \& Gelino, D. M. 2011, ApJ, 729, 74

Kane, S. R., \& Gelino, D. M. 2012a, MNRAS, 424, 779

Kane, S. R., \& Gelino, D. M. 2012b, PASP, 124, 323

Kane, S. R., \& Gelino, D. M. 2012c, AsBio, 12, 940

Kasting, J. F., Whitmire, D. P., \& Reynolds, R. T. 1993, Icar, 101, 108

Kipping, D. M., Forgan, D., Hartman, J., et al. 2013, ApJ, 777, 134

Kopparapu, R. K., Ramirez, R., Kasting, J. F., et al. 2013, ApJ, 765, 131

Kopparapu, R. K., Ramirez, R. M., SchottelKotte, J., et al. 2014, ApJL, 787, L29

Lovis, C., Ségransan, D., Mayor, M., et al. 2011, A\&A, 528, 112

Marcy, G. W., Isaacson, H., Howard, A. W., et al. 2014, ApJS, 210, 20

McArthur, B. E., Endl, M., Cochran, W. D., et al. 2004, ApJL, 614, L81

Perryman, M. A.C., Lindegren, L., Kovalevsky, J., et al. 1997, A\&A, 323, L49

Seager, S., Kuchner, M., Hier-Majumder, C. A., \& Militzer, B. 2007, ApJ, 669,1279

Torres, G., Andersen, J., \& Giménez, A. 2010, A\&ARv, 18, 67

Tuomi, M. 2012, A\&A, 543, 52

Tuomi, M., Kotiranta, S., \& Kaasalainen, M. 2009, A\&A, 494, 769

van Leeuwen, F. (ed.) 2007, in Hipparcos, the New Reduction of the Raw Data (Astrophysics and Space Science Library, Vol. 350; Berlin: Springer)

Wang, S. X., Wright, J. T., Cochran, W., et al. 2012, ApJ, 761, 46

Weiss, L. M., \& Marcy, G. W. 2014, ApJL, 783, L6

Weiss, L. M., Marcy, G. W., Rowe, J. F., et al. 2013, ApJ, 768, 14

Wiktorowicz, S. J. 2009, ApJ, 696, 1116

Williams, D. M., \& Pollard, D. 2002, IJAsB, 1, 61

Wisdom, J. 2006, AJ, 131, 2294

Wisdom, J., \& Holman, M. 1991, AJ, 102, 1528

Wright, J. T., Upadhyay, S., Marcy, G. W., et al. 2009, ApJ, 693, 1084 